\title{
Novel sub-eV high throughput laboratory micro-XAS (x-ray absorption spectrometer) for chemical state analysis through XANES and EXAFS
}

\author{
Wenbing Yun ${ }^{1}$, Srivatsan Seshadri ${ }^{1}$, Sylvia Lewis ${ }^{1}$, Jeff Gelb ${ }^{1}$, SH Lau ${ }^{1}$, and Janos Kirz ${ }^{1,2}$ \\ 1. Sigray, Inc., Concord, CA, USA \\ 2. Lawrence Berkeley National Laboratory, Berkeley, CA, USA
}

X-ray absorption spectrometry (XAS) is a commonly used synchrotron-based technique that enables determination of the chemical state of an element of interest, including oxidation state, local atomic geometry, bond lengths, and coordination number. The technique has been applied for a substantial range of applications, including mineralogic determination in geosciences, chemical states of nanoparticles, catalysts, and battery lithiation processes in operando.

There is immense interest in enabling fast throughput XAS in the laboratory. Until more recently, this effort has largely been hampered by the limited flux of x-ray source at the energies required [1] and by the energy resolution achievable [2]. Advances in x-ray source technology and in curved crystal spectrometer designs, in tandem with significant improvements to x-ray detectors with small pixel sizes, has enabled several new viable designs for laboratory XAS in the academic community.

Here we present a novel commercial laboratory XAS with substantial improvements over leading designs. The compact XAS system operates in the 4-10 keV energy range and enables micro-XAS capabilities with a resolution of 10 micrometers. The system utilizes a patented microstructured x-ray source coupled with an innovative x-ray optic design, which provides major performance advantages including operation of the x-ray source at higher efficiencies, high collection efficiency, and enabling smaller focal spots. Downstream, multiple sagitally curved mosaic and single crystals are used as analyzers with a direct detection CCD detector.

The system enables high energy resolution at sub-eV and at high throughput, for collection of data within a single exposure of approximately $1 \mathrm{keV}$ from several seconds to a few minutes with no contamination from higher order reflections. The fast acquisition times enable a wide variety of potential applications that include in situ and in operando measurements, quick EXAFS and others. In this talk, we will discuss the basic principles behind the design of the instrument and its performance. 


\section{References:}

[1] K Sakurai and X Guo, Spectrochemica Acta B: Atomic Spectroscopy 54 (1999), p. 99-107.

[2] GT Seidler, DR Mortensen, AJ Remesnik, JI Pacold, NA Ball, N Barry, M Styczinski, OR Hoidn, Review of Scientific Instruments 85 (2014).

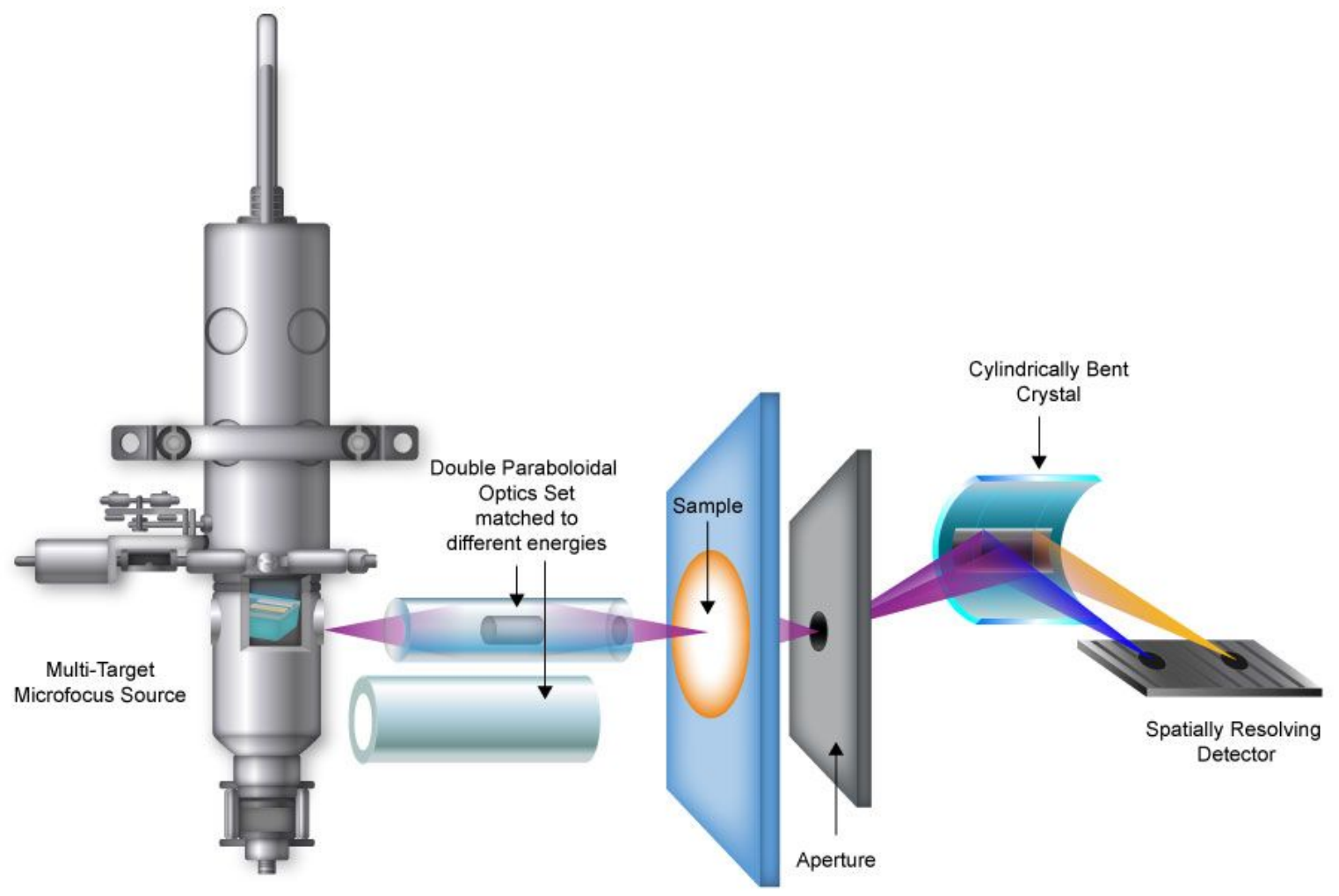

Figure 1. The x-ray absorption spectrometer design (XAS) comprises of innovations in the x-ray source and optic technology to enable high spectral and spatial resolutions at high throughput 